\title{
ON THE DIAGONAL OF AN OPERATOR
}

BY

\author{
PENG FAN
}

\begin{abstract}
Characterizations of zero-diagonal operators (i.e., operators that have a diagonal whose entries consist entirely of zeros) and the norm-closure of these operators are obtained. Also included are new characterizations of trace class operators, self-commutators of bounded operators, and others.
\end{abstract}

0. Introduction. In [3], it is shown that a hermitian compact operator is a self-commutator of a compact operator if and only if it has a zero-diagonal. Motivated by this result, we decided to launch an investigation into the connection between the diagonal of an operator and the operator itself; we wonder to what extent an operator can be described by its diagonal. It turns out that, as expected, the diagonal of an operator carries more information about the operator than its relatively small size (compare to the "fat" matrix representation of the operator) may suggest.

Let $H$ be a (complex, separable) Hilbert space, and $B(H)$ the space of all (bounded) operators on $H$. By zero-diagonal operators we mean the operators $T$ such that there exists an orthonormal basis $\left\{b_{j}\right\}$ for $H$ satisfying $\left(T b_{j}, b_{j}\right)=0$ for all $j$. It is not hard to see that the set of these operators is pathwise connected (in fact, all its elements can be connected to the origin 0 inside itself); that it is not a linear manifold $(2 I \oplus(-I)$ and $(-I) \oplus 2 I$ belong to it but their sum $I \oplus I$ does not $)$; and moreover, that it stays away from $I$. (Indeed, the distance is no less than one: Take a zero-diagonal operator $T$ and choose an orthonormal basis $\left\{b_{j}\right\}$ so that $\left(T b_{j}, b_{j}\right)=0$. Then $\|T-I\|=\sup _{\|x\| \leqslant 1}((T-I) x, x) \geqslant\left((T-I) b_{j}, b_{j}\right)=1$.)

In the first section we are to characterize zero-diagonal operators and hermitian zero-diagonal operators.

$\$ 2$ is devoted to the identification of the norm-closure of zero-diagonal operators. It turns out that this is a quite well-known class of operators. As a result, we are able to obtain new characterizations for some related classes of operators-among them, self-commutators of bounded operators and trace class operators.

1. Zero-diagonal operators. Let $H$ be a finite-dimensional Hilbert space and $T$ an operator acting on $H$. Then the following three statements are equivalent:

(1) $T$ has a zero-diagonal;

Received by the editors May 19, 1981 and, in revised form, May 3, 1983.

1980 Mathematics Subject Classification. Primary 47A12, 47B99; Secondary 47B10, 47B47.

Key words and phrases. Zero-diagonal operators, trace class operators, self-commutators.

' This paper is part of the author's dissertation written under the direction of Professor J. G. Stampfli. The author wishes to thank Professor Stampfli for his encouragement and guidance. 
(2) $\operatorname{Tr}(T)=0$;

(3) $T=A B-B A$, where $A$ and $B$ belong to $B(H)$.

This can be seen in [7, p. 109]. Supposing $H$ is infinite-dimensional, things become substantially different. For one thing, $\operatorname{Tr}(T)$ may not make sense any more even if rearrangement is taken into account; for another, $A B-B A$ can be anything except $\lambda I+K$, where $\lambda$ is a complex number and $K$ a compact operator, by a theorem of Brown and Pearcy [2]. Thus finding a weaker alternative for the characterization of zero-diagonal operators now seems inevitable. Fortunately, statement (2) can be modified to achieve such a task.

THEOREM 1. The necessary and sufficient condition that an operator $T$ has a zero-diagonal is that there exists an orthonormal basis $\left\{e_{n}\right\}$ such that $\left\{s_{n}\right\} \rightarrow 0$ as $j \rightarrow \infty$, where $s_{n}=\sum_{k=1}^{n}\left(T e_{k}, e_{k}\right)$ and $\left\{s_{n}\right\}$ is a subsequence of $\left\{s_{n}\right\}$.

Corollaries 1 and 2, whose proofs are omitted, are also independently obtained by J. P. Williams ${ }^{2}$ and others, using Zorn's lemma and a trace argument; they were first proved in [4] for matrices.

COROLlaRY 1. Let $T$ be a trace class operator. Then $T$ has a zero-diagonal if and only if $\operatorname{Tr}(T)=0$.

COROLlaRY 2. Under the same hypothesis, $T$ has a main diagonal $(\operatorname{Tr}(T), 0,0, \ldots)$ if and only if $\operatorname{Tr}(T) \in W(T)$, the numerical range of $T$.

Replacing unitary equivalence by similarity, we obtain the following proposition which was again first proved in [4] for matrices but whose proof applies to trace class operators as well. We include it for completeness.

Proposition. Let $T$ be a trace class operator. Then $T$ is similar to a trace class operator of the form

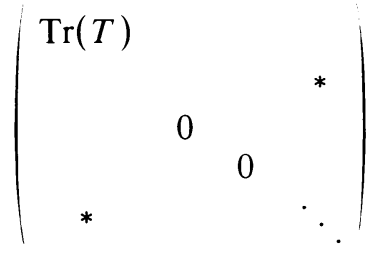

if and only if $T$ is not a scalar.

Proof. If $T$ is not a scalar, then there exists a unit vector $x$ such that $x$ and $T x$ are linear independent and a vector $z$ independent of $x$ such that $(z, x)=\operatorname{Tr}(T)$. Define

$$
S:\left\{\begin{array}{l}
x \mapsto x, \\
z \mapsto T x \\
\{x, z\}^{\perp} \mapsto\{x, T x\}^{\perp} \quad \text { unitarily. }
\end{array}\right.
$$

Then $\left(S^{-1} T S x, x\right)=(z, x)=\operatorname{Tr}(T)$. Since $\operatorname{Tr}(T)=\operatorname{Tr}\left(S^{-1} T S\right)$, one can find $e_{j}$, $j=1,2, \ldots$, such that $\left(S^{-1} T S e_{j}, e_{j}\right)=0$ by Theorem 1 . This completes the proof.

${ }^{2}$ Private communication. 
Letting $W^{0}(T)$ denote the interior of $W(T)$, the numerical range of $T$ (for a definition, see [8]) in $C$, we arrive at the following corollary, whose proof is omitted.

COROllary 3. If $W^{0}(T)$ is nonempty and if $T$ is a zero-diagonal operator, then $0 \in W^{0}(T)$. The converse is not true.

The next theorem was first proved in [3] for compact hermitian operators.

THEOREM 2. Let $T$ be hermitian operator and write $T=T^{+} \oplus\left(-T^{-}\right)$, where $T^{+}$ and $T^{-}$are positive. Then $T$ has a zero-diagonal if and only if $\operatorname{Tr}\left(T^{+}\right)=\operatorname{Tr}\left(T^{-}\right) \leqslant \infty$.

An immediate consequence of this theorem is the following corollary, whose proof is again omitted.

Corollary. Let $T$ be a hermitian operator. If $0 \in W_{e}^{0}(T)$, the interior of the essential numerical range of $T$ in $R$ (see [6] for a complete definition), then $T$ has a zero-diagonal. Further $\lambda \in W_{e}^{0}(T)$ implies $T$ has a $\lambda$-diagonal (that is, there exists an orthonormal basis $\left\{e_{j}\right\}$ such that $\left(T e_{j}, e_{j}\right)=\lambda$ for all $\left.j\right)$.

We now prove Theorem 1, which needs these lemmas: Lemma 4 will be used to produce unit vectors, with respect to which the diagonal entries of the matrix representation of the operator $T$ are zeros, while Lemmas 1-3 serve to insure that these unit vectors form an orthonormal basis.

LeMma 1. Let $\left\{b_{j}\right\}_{j=1}^{\infty}$ be an orthonormal set, let $\Re_{n}=V\left\{b_{j}\right\}_{j=1}^{n}$, and let $P_{n}$ be the projection on $\mathfrak{\pi}_{n}$. If $P_{n} \rightarrow I$ strongly (in fact, a subsequence of $P_{n}$ is enough), then $\left\{b_{j}\right\}_{j=1}^{\infty}$ forms a basis.

Proof. Suppose not; there exists $0 \neq x \in H$ such that $x \perp V\left\{b_{j}\right\}_{j=1}^{\infty}$. This implies $P_{n} x=0$ for all $n$; hence $P_{n} \rightarrow I$ strongly. This contradicts the hypothesis.

LEMMA 2. Let $\left\{a_{j}\right\}_{j=1}^{\infty}$ be a sequence of positive numbers such that $\sum_{j=1}^{\infty} a_{j}<\infty$ and let $s_{n}^{\prime}=\sum_{j=n}^{\infty} a_{j}$. Then $\sum_{n=1}^{\infty}\left(a_{n} / s_{n}^{\prime}\right)=\infty$.

PROOF. Elementary calculations.

REMARK. The hypothesis of Lemma 2 can be substantially weakened. Precisely, if $\left\{a_{j}\right\}$ is a sequence of real numbers and if a subsequence of $s_{n}^{\prime}$ approaches 0 , then the conclusion still holds.

LeMma 3. Let

$$
T=\left(\begin{array}{cc}
d_{1} & z \\
z^{\prime} & d_{2}
\end{array}\right)
$$

be a matrix over the field of complex numbers, with respect to an orthonormal basis $\left\{e_{1}, e_{2}\right\}$, and let $d$ lie on the line segment connecting $d_{1}$ and $d_{2}$. Then there exists a unit vector $f$ such that $(T f, f)=d$ and $\left|\left(e_{1}, f\right)\right| \leqslant \sqrt{\left(d_{2}-d\right) /\left(d_{2}-d_{1}\right)}$.

Proof. Without loss, we can assume that $d=0, d_{1}>0$, and $d_{2}<0$. Indeed, if we write

$$
T=e^{i \theta}\left[e^{-i \theta}(T-d)\right]+d
$$


where $\theta=\arg \left(d_{1}-d\right)$, then $T^{\prime}=e^{-i \theta}(T-d)$ has the properties we need; thus, if we can choose a unit vector $f$ such that $\left(T^{\prime} f, f\right)=0$ and

$$
\left|\left(e_{1}, f\right)\right| \leqslant \sqrt{\frac{e^{-i \theta}\left(d_{2}-d\right)-0}{e^{-i \theta}\left[\left(d_{2}-d\right)-\left(d_{1}-d\right)\right]}},
$$

one sees that $(T f, f)=d$ and $\left|\left(e_{1}, f\right)\right| \leqslant \sqrt{\left(d_{2}-d\right) /\left(d_{2}-d_{1}\right)}$.

Now, let us start the required calculation. We are to find a number $c(=a+i b)$ such that $\left(T\left(c e_{1}+e_{2}\right), c e_{1}+e_{2}\right)=0$. Since

$$
\begin{aligned}
\left(T\left(c e_{1}+e_{2}\right), c e_{1}\right. & \left.+e_{2}\right)=\left(c d_{1} e_{1}+c z^{\prime} e_{2}+z e_{1}+d_{2} e_{2}, c e_{1}+e_{2}\right) \\
& =|c|^{2} d_{1}+\bar{c} z+c z^{\prime}+d_{2} \\
& =|c|^{2} d_{1}+d_{2}+a x+b y+a x^{\prime}-b y^{\prime}+i\left(a y-b x+a y^{\prime}+b x^{\prime}\right),
\end{aligned}
$$

where $z=x+i y$ and $z^{\prime}=x^{\prime}+i y^{\prime}$, it follows that

$$
|c|^{2} d_{1}+d_{2}+a\left(x+x^{\prime}\right)+b\left(y-y^{\prime}\right)=0
$$

and

$$
a\left(y+y^{\prime}\right)-b\left(x-x^{\prime}\right)=0
$$

To make the estimation easier, we break it into two cases.

Case (i). When $x=x^{\prime}$, choose $a=0$. Then $(*)$ becomes $b^{2} d_{1}+d_{2}+b\left(y-y^{\prime}\right)$; hence

$$
b=\frac{-\left(y-y^{\prime}\right) \pm \sqrt{\left(y-y^{\prime}\right)^{2}-4 d_{1} d_{2}}}{2 d_{1}} .
$$

Thus we obtain the inequality

$$
\begin{aligned}
|c|^{2} & =a^{2}+b^{2}=\frac{2\left(y-y^{\prime}\right)^{2} \pm 2\left(y-y^{\prime}\right) \sqrt{\left(y-y^{\prime}\right)^{2}-4 d_{1} d_{2}}-4 d_{1} d_{2}}{4 d_{1}^{2}} \\
& \leqslant \frac{-4 d_{1} d_{2}}{4 d_{1}^{2}}=-\frac{d_{2}}{d_{1}} .
\end{aligned}
$$

Case (ii). When $x \neq x^{\prime}$, choose $b$ so that

$$
\frac{b}{a}=\frac{y+y^{\prime}}{x-x^{\prime}}=k
$$

Substituting into $(*)$, we obtain

$$
a^{2} d_{1}\left(1+k^{2}\right)+d_{2}+a\left(x+x^{\prime}\right)+a k\left(y-y^{\prime}\right)=0 ;
$$

hence

$$
a=\frac{-\left(x+x^{\prime}+k y-k y^{\prime}\right) \pm \sqrt{\left(x+x^{\prime}+k y-k y^{\prime}\right)^{2}-4 d_{1} d_{2}\left(1+k^{2}\right)}}{2 d_{1}\left(1+k^{2}\right)}
$$


thus

$$
\begin{aligned}
a^{2}= & \frac{2\left(x+x^{\prime}+k y-k y^{\prime}\right)^{2}-4 d_{1} d_{2}\left(1+k^{2}\right)}{4 d_{1}^{2}\left(1+k^{2}\right)^{2}} \\
& \frac{ \pm 2\left(x+x^{\prime}+k y-k y^{\prime}\right) \sqrt{\left(x+x^{\prime}+k y-k y^{\prime}\right)^{2}-4 d_{1} d_{2}\left(1+k^{2}\right)}}{4 d_{1}^{2}\left(1+k^{2}\right)^{2}} \\
\leqslant & \frac{-4 d_{1} d_{2}\left(1+k^{2}\right)}{\not 4 d_{1}^{2}\left(1+k^{2}\right)^{2}}=\frac{-d_{2}}{d_{1}\left(1+k^{2}\right)} .
\end{aligned}
$$

Therefore, $|c|^{2}=a^{2}+b^{2}=a^{2}\left(1+k^{2}\right) \leqslant-d_{2} / d_{1}$.

Putting these cases together and letting $f=\left(c e_{1}+e_{2}\right)\left(1+|c|^{2}\right)^{-1 / 2}$, we see that

$$
(T f, f)=\left(1+|c|^{2}\right)^{-1}\left(T\left(c e_{1}+e_{2}\right), c e_{1}+e_{2}\right)=0,
$$

and that

$$
\left(e_{1}, f\right)=\frac{|c|}{\sqrt{1+|c|^{2}}}=\left(1+|c|^{-2}\right)^{-1 / 2} \leqslant\left(1-d_{1} d_{2}^{-1}\right)^{-1 / 2}=\sqrt{\frac{d_{2}-0}{d_{2}-d_{1}}} .
$$

These conclude our proof.

The following lemma can be found in many linear algebra books (see, e.g., [7, p. 109]).

LEMMA 4. Let $T$ be a square matrix over the field of complex numbers. Then $T$ has a zero-diagonal if and only if $\operatorname{Tr}(T)=0$.

Proof of Theorem 1. Let $d_{j}=\left(T e_{j}, e_{j}\right)$ for $j=1,2, \ldots$ According to the way the entries (counting multiplicities) of the diagonal $\left\{d_{j}\right\}_{j=1}^{\infty}$ are distributed on the plane, the proof will be divided into three parts.

PART I. For every line passing through the origin, there are infinitely many diagonal entries (counting multiplicities) lying on each of the (open) half-planes divided by the line.

By the assumption, it is easy to see that we can find three diagonal entries say, $d_{1}, d_{2}$, and $d_{3}$ such that the origin lies in the interior of the triangle formed by these three points. Since $\left\{S_{n_{j}}\right\}$ approaches zero as $j$ goes to infinity, we can secure an element, say, $S_{n_{1}}$ lying in the interior of the triangle; so we can have a unit vector $f$, which is a linear combination of $e_{1}, e_{2}$, and $e_{3}$, satisfying $(T f, f)=S_{n_{1}}$ (numerical range is convex, see [8, p. 110]); hence in the orthogonal complement of $f$ with respect to $V\left\{e_{1}, e_{2}, \ldots, e_{n_{1}}\right\}$, we can produce an orthonormal set $\left\{b_{1}, b_{2}, \ldots, b_{n_{1}-1}\right\}$ such that $\left(T b_{j}, b_{j}\right)=0$ for $j=1,2, \ldots, n_{1}-1$ (by Lemma 4). In the same fashion, we can find, say, $d_{n_{1}+1}, d_{n_{1}+2}$, and $d_{n_{1}+3}$ such that the origin and, say, $S_{n_{2}}$ are enclosed in the interior of the triangle formed by these points; again we can produce an orthonormal set $\left\{b_{n_{1}}, \ldots, b_{n_{2}-1}\right\}$ and a unit vector $g$, a linear combination of $e_{n_{1}+1}, e_{n_{1}+2}$, and $e_{n_{1}+3}$, such that $\left(T b_{j}, b_{j}\right)=0$ for $j=n_{1}, \ldots, n_{2}-1$ and $(T g, g)=$ $S_{n_{2}}$. Continuing this process, we can produce an orthonormal set $\left\{b_{j}\right\}_{j=1}^{\infty}$ satisfying $V\left\{e_{j}\right\}_{j=1}^{n_{k}} \subseteq V\left\{b_{j}\right\}_{j=1}^{n_{k+1}}$; this shows, by Lemma 1 , that $\left\{b_{j}\right\}_{j=1}^{\infty}$ forms a basis. 
PART II. For every line passing through the origin, there are infinitely many diagonal entries (counting multiplicities) not lying on the line, but, for at least one such line, there are only finitely many entries lying one one of the half-planes divided by this line.

Without loss, we can suppose that the line is the $x$-axis and that only one point, say, $d_{1}$ lies on the upper half-plane and the rest on the (closed) lower plane. As in Part I, we can find, say, $d_{2}$ and $d_{3}$ such that the origin lies in the interior of the triangle formed by $d_{1}, d_{2}$, and $d_{3}$. Since $-\sum_{j=2}^{\infty} \operatorname{Im} d_{j}=\operatorname{Im} d_{1}$, it is not hard to see that we can pick, say, $s_{n_{1}}$ so small that $\left|t_{1}-s_{n_{1}}\right| \leqslant \frac{2}{3}\left|t_{1}-d_{1}\right|$, where $t_{1}$ is the intersection of the line segment connecting $d_{2}$ and $d_{3}$ and the line passing through $d_{1}$ and $s_{n_{1}}$. (Here, of course, $n_{1} \geqslant 3$ and $s_{n_{1}}$ lies between $d_{1}$ and $t_{1}$.) Thus, by Lemma 3, there is a unit vector $f_{1}$ such that $\left(T f_{1}, f_{1}\right)=s_{n_{1}}$ and

$$
\left|\left(e_{1}, f_{1}\right)\right| \leqslant \sqrt{\frac{t_{1}-s_{n_{1}}}{t_{1}-d_{1}}} \leqslant \frac{2}{3}
$$

( $f_{1}$ is a linear combination of $e_{1}, e_{2}$, and $\left.e_{3}\right)$; meantime, by Lemma 4, we can find, in the orthogonal complement of $f_{1}$ with respect to $V\left\{e_{1}, \ldots, e_{n_{1}}\right\}$, an orthogonal set $\left\{b_{1}, \ldots, b_{n_{1}-1}\right\}$ with $\left(T b_{j}, b_{j}\right)=0$ for $j=1, \ldots, n_{1}-1$.

Following the same line of argument, we can pick $d_{n_{1}+1}, d_{n_{1}+2}$, and small enough $s_{n_{2}}$ satisfying $\left|t_{2}-s_{n_{2}}\right| \leqslant \frac{2}{3}\left|t_{2}-s_{n_{1}}\right|$, where $t_{2}$ is the intersection of the line segment connecting $d_{n_{1}+1}$ and $d_{n_{1}+2}$ and the line passing through $s_{n_{1}}$ and $s_{n_{2}}$. Thus, as before, there are mutually orthogonal unit vectors $b_{n_{1}}, \ldots, b_{n_{2}-1}$ and $f_{2}$ such that $\left(T b_{j}, b_{j}\right)=0$. for $j=n_{1}, \ldots, n_{2}-1$ and $\left(T f_{2}, f_{2}\right)=s_{n_{2}}$ and

$$
\left|\left(f_{1}, f_{2}\right)\right| \leqslant \sqrt{\frac{t_{2}-s_{n_{2}}}{t_{2}-s_{n_{1}}}} \leqslant \frac{2}{3} \text {. }
$$

( $f_{2}$ is a linear combination of $f_{1}, e_{n_{1}+1}$ and $e_{n_{1}+2}$.) Continuing this procedure, eventually we are able to manufacture an orthonormal set $\left\{b_{j}\right\}_{j=1}^{\infty}$ satisfying $\left(T b_{j}, b_{j}\right)$ $=0$ for all $j$ and $\left\{f_{j}\right\}_{j=1}^{\infty}$ satisfying $\left(T f_{j}, f_{j}\right)=s_{n}$, and $\left|\left(f_{j-1}, f_{j}\right)\right| \leqslant \frac{2}{3}$ for all $j$, where $f_{0}=e_{1}$. $\left(f_{j}\right.$ is a linear combination of $f_{j-1}, e_{n_{j-1}+1}$, and $e_{n_{j-1}+2}$ for all $j$, where $n_{0}=1$; the orders of the basis and the subsequence may have been rearranged, for neatness' sake.) Further,

$$
\begin{aligned}
\left|\left(e_{j}, f_{j+k}\right)\right| \leqslant \mid\left(e_{j},\left(f_{j+k}, f_{j+k-1}\right) f_{j+k-1}+\left(f_{j+k}, e_{n_{j+k-1}+1}\right) e_{n_{j+k-1}+1}\right. & \left.\quad+\left(f_{j+k}, e_{n_{j+k-1}+2}\right) e_{n_{j+k-1}+2}\right) \mid \\
& =\left|\left(e_{j},\left(f_{j+k}, f_{j+k-1}\right) f_{j+k-1}\right)\right| \\
& =\left|\left(e_{j}, f_{j+k-1}\right)\right| \cdot\left|\left(f_{j+k-1}, f_{j+k}\right)\right| \\
& \leqslant\left|\left(e_{j}, f_{j}\right)\right| \cdot\left|\left(f_{j}, f_{j+1}\right)\right| \cdots\left|\left(f_{j+k-1}, f_{j+k}\right)\right| \\
& \leqslant\left|\left(e_{j}, f_{j}\right)\right| \cdot\left(\frac{2}{3}\right)^{k} .
\end{aligned}
$$


This implies $\left(e_{j}, f_{j+k}\right) \rightarrow 0$ as $k \rightarrow \infty$. Since

$$
\left\|\left(I-P_{n_{, k}-1}\right) e_{j}\right\|^{2}=\left\|Q_{j+k} e_{j}\right\|^{2}=\left|\left(e_{j}, f_{j+k}\right)\right|^{2},
$$

where $Q_{j+k}=$ projection on $V\left\{f_{j+k}, e_{n_{j+k}+1}, \ldots\right\}$, this shows $\left\{b_{j}\right\}_{j=1}^{\infty}$ forms a basis by Lemma 1 .

PART III. For at least one line passing through the origin, there are only finitely many diagonal entries (counting multiplicities) not lying on the line.

We may assume that all $d_{j}$ 's are on the $x$-axis. (In fact, rotate the line in alignment with the $x$-axis if necessary; as for the exposed points, $s_{n}$ will be real as soon as $n$ is big enough.) There are two cases to consider.

Case (i). There are infinitely many diagonal entries (counting multiplicities) lying on each side of the origin. The proof is analogous to Part I.

Case (ii). There are infinitely many diagonal entries (counting multiplicities) lying on one side of the origin and only finitely many on the other. Without loss, we can assume that $d_{1}>0$ and $d_{j}<0$ for $j=2,3, \ldots$; then let $f_{1}=e_{1}$. Similar to Part II, choose a unit vector $f_{2} \in V\left\{e_{1}, e_{2}\right\}$ so that $\left(T f_{2}, f_{2}\right)=s_{2}\left(=d_{1}+d_{2}\right)$, and

$$
\left|\left(e_{1}, f_{2}\right)\right| \leqslant \sqrt{\frac{d_{2}-\left(d_{1}+d_{2}\right)}{d_{2}-d_{1}}}=\sqrt{\frac{d_{1}}{d_{1}-d_{2}}}=\left(1-d_{2} d_{1}^{-1}\right)^{-1 / 2}
$$

(by Lemma 3); then, take another unit vector $b_{1} \in V\left\{e_{1}, e_{2}\right\}$ orthogonal to $f_{2}$-clearly $\left(T b_{1}, b_{1}\right)=0$. (Note $f_{2}$ is a linear combination of $f_{1}$ and $e_{2}$.)

In the same manner, we can find by induction an orthonormal set $\left\{b_{1}, b_{2}, \ldots\right\}$ and unit vectors $f_{1}, f_{2}, \ldots$ such that $\left(T b_{j}, b_{j}\right)=0,\left(T f_{j}, f_{j}\right)=s_{j}\left(=\sum_{k=1}^{j} d_{k}\right)$, and

$$
\left(f_{j}, f_{j+1}\right) \leqslant\left[1-d_{j+1}\left(d_{1}+\cdots+d_{j}\right)^{-1}\right]^{-1 / 2}
$$

for $j=1,2, \ldots\left(f_{j+1}\right.$, orthogonal to $\left\{b_{1}, \ldots, b_{j}\right\}$, is a linear combination of $f_{j}$ and $e_{j+1}$ for all $j$.) Moreover,

$$
\begin{aligned}
\left|\left(e_{j}, f_{j+k}\right)\right| & =\left|\left(e_{j},\left(f_{j+k}, f_{j+k-1}\right) f_{j+k-1}+\left(f_{j+k}, e_{j+k}\right) e_{j+k}\right)\right| \\
& =\left|\left(e_{j}, f_{j+k-1}\right) \cdot\left(f_{j+k-1}, f_{j+k}\right)\right| \\
& =\left|\left(e_{j}, f_{j}\right) \cdot\left(f_{j}, f_{j+1}\right) \cdots\left(f_{j+k-1}, f_{j+k}\right)\right| \\
& \leqslant\left[\left(1-d_{j+1} s_{j}^{-1}\right) \cdots\left(1-d_{j+k} s_{j+k-1}^{-1}\right)\right]^{-1 / 2} \\
& =\left[\left(1+d_{j+1} s_{j+1}^{\prime-1}\right) \cdots\left(1+d_{j+k} s_{j+k}^{\prime-1}\right)\right]^{-1 / 2}, \\
& =\prod_{n=j+1}^{j+k}\left(1+\frac{d_{n}}{s_{n}^{\prime}}\right),
\end{aligned}
$$

where $s_{n+1}^{\prime}=\sum_{j=n+1}^{\infty} d_{j}=\sum_{j=1}^{n} d_{j}=-s_{n}$.

To complete the proof, we need to show $\left\{b_{j}\right\}_{j=1}^{\infty}$ forms a basis; it is enough, utilizing Lemma 1 , to prove that $\left\|\left(I-P_{j+k}\right) e_{j}\right\| \rightarrow 0$ as $k \rightarrow \infty$ for all $j$ where $P_{j+k}=$ Proj on $V\left\{b_{1}, \ldots, b_{j+k}\right\}$. Since, by Lemma 2, $\sum_{n=1}^{\infty} d_{n} / s_{n}^{\prime}=\infty$ (note that 
$d_{n} / s_{n}^{\prime}>0$ for $n \geqslant 2$ ), one sees that

$$
\prod_{n=j+1}^{j+k}\left(1+\frac{d_{n}}{s_{n}^{\prime}}\right) \rightarrow \infty \text { as } k \rightarrow \infty ;
$$

and since $\left\|\left(I-P_{j+k-1}\right) e_{j}\right\|^{2}=\left\|Q_{j+k} e_{j}\right\|^{2}=\left|\left(e_{j}, f_{j+k}\right)\right|^{2}$, where $Q_{j+k}=$ Proj on $V\left\{f_{j+k}, e_{j+k+1}, \ldots\right\}$, one has that $\left\|\left(I-P_{j+k-1}\right) e_{j}\right\| \rightarrow 0$ as $k \rightarrow \infty$ for all $j$. And so the proof of Theorem 1 is complete.

Proof of Theorem 2. Necessity. Suppose that $\left\{b_{j}\right\}$ is an orthonormal basis such that $\left(T b_{j}, b_{j}\right)=0$ for all $j$. Since $T^{+} \oplus 0=T+\left(0 \oplus T^{-}\right)$,

$$
\begin{aligned}
\operatorname{Tr}\left(T^{+}\right) & =\operatorname{Tr}\left(T^{+} \oplus 0\right)=\left(\left(T^{+} \oplus 0\right) b_{j}, b_{j}\right) \\
& =\Sigma\left(\left(T b_{j}, b_{j}\right)+\left(\left(0 \oplus T^{-}\right) b_{j}, b_{j}\right)\right) \\
& =\Sigma\left(\left(0 \oplus T^{-}\right) b_{j}, b_{j}\right)=\operatorname{Tr}\left(0 \oplus T^{-}\right)=\operatorname{Tr}\left(T^{-}\right) .
\end{aligned}
$$

Sufficiency. If $\operatorname{Tr}\left(T^{+}\right)=\operatorname{Tr}\left(T^{-}\right)<\infty$, then $T$ has a zero-diagonal (by the theorem in [3]). So, suppose that $\operatorname{Tr}\left(T^{+}\right)=\operatorname{Tr}\left(T^{-}\right)=\infty$ and that $T^{+}$and $T^{-}$are positive definite. The idea of the proof is to rearrange the entries so that the condition of Theorem 1 is satisfied. However, such rearrangement may not exist (e.g., let the diagonal entries be $\left.\left\{\frac{1}{2}, 1,1, \ldots ;-1,-1, \ldots\right\}\right)$. Thus, a modification job of the diagonal entries is indispensable.

Let $\left\{e_{m}\right\}$ and $\left\{f_{n}\right\}$ be orthonormal basis for $H^{+}$and $H^{-}$, respectively, and let $p_{m}=\left(T^{+} e_{m}, e_{m}\right)$ and $q_{n}=\left(T^{-} f_{n}, f_{n}\right)$. We will divide the rest of the proof into two steps.

Step I. Suppose $\left\{p_{m}\right\}$ (or $\left\{q_{n}\right\}$ ) has a subsequence, converging to zero, whose summation is equal to infinite. Then it is not hard to rearrange the sequence of the diagonal entries so that the hypothesis of Theorem 1 is satisfied.

Step II. Suppose the hypothesis in Step I is not met. Then $\left\{p_{m}\right\}$ has a positive limit point, say, 2. We shall manufacture a sequence of positive entries that satisfies the hypothesis of Step I.

Let $\left\{p_{m_{1}}\right\}$ be a subsequence of $\left\{p_{m}\right\}$, converging to 2 , such that $\left|p_{m_{1}}-2\right|<1$ and $\left\{q_{n}\right\}$ a subsequence of $\left\{q_{n}\right\}$ such that $\Sigma_{n \neq n}, q_{n}=\infty$. For each pair $p_{m}$, and $-q_{n}$ $(j=1,2, \ldots)$, we can produce a new pair of entries $1 / j$ and $p_{m_{1}}-q_{n_{1}}-1 / j$ since $p_{m}>1 / j>-q_{n}$, and the fact that the numerical range is convex. The subsequences $\{1 / j\}$ and $\left\{q_{n}\right\}_{n \neq n}$, guarantee that the hypothesis of Step I is satisfied, and this completes the proof.

2. The norm-closure of zero-diagonal operators. The norm-closure of the set of zero-diagonal operators seems enormous: weighted shifts, quasinilpotent operators, compact operators, and, of course, those satisfying the condition of Theorem 1 are all members of this set. Just how big is this set? Is this class of operators closed under similarity? These questions will be answered following the characterization of this set. We shall then shift our attention to two different classes of operators: self-commutators and trace class operators. Our goal: to give each of them a new characterization.

THEOREM 3. The necessary and sufficient condition that an operator $T$ is a member of the norm-closure of the set of zero-diagonal operators is that $0 \in W_{e}(T)$, the essential numerical range of $T$. 
Proof. Necessity. By hypothesis, there exists a sequence of zero-diagonal operators $\left\{T_{n}\right\}_{n=1}^{\infty}$ such that $\left\|T-T_{n}\right\| \leqslant 1 / n$ for $n=1,2, \ldots$. Since for each $T_{n}, n=$ $1,2, \ldots$, there corresponds an orthonormal basis $B^{(n)}=\left\{b_{j}^{(n)}\right\}_{j=1}^{\infty}$ such that $\left(T_{n} b_{j}^{(n)}, b_{j}^{(n)}\right)=0$ for all $n$ and all $j$, one sees that $\left|\left(T b_{j}^{(n)}, b_{j}^{(n)}\right)\right| \leqslant 1 / n$ for all $n$ and all $j$. Pick an element $b_{j}$ from each basis $B^{(j)}$ satisfying $\left|\left(b_{j}, b_{k}\right)\right| \leqslant 1 / 2^{j}$ for all $1 \leqslant k<j$ (by induction); it follows that $b_{j} \rightarrow 0$ weakly, and $\left|\left(T b_{j}, b_{j}\right)\right| \leqslant 1 / j \rightarrow 0$ as $j \rightarrow \infty$. By [6, Theorem (5.1)], $0 \in W_{e}(T)$.

Sufficiency. Suppose $0 \in W_{e}(T)$. Again by [6, Theorem (5.1)], there exists an infinite-dimensional projection $P$ such that $P T P$ is compact; thus $P T P$ can be perturbed by a small operator $\varepsilon$ so that $P T P-\varepsilon$ is a zero-diagonal operator. That is,

$$
\begin{aligned}
& T=\left(\begin{array}{cc}
* & * \\
* & P T P
\end{array}\right)=\left(\begin{array}{cc}
* & * \\
* & P T P-\varepsilon
\end{array}\right)+\left(\begin{array}{ll}
0 & 0 \\
0 & \varepsilon
\end{array}\right) \\
& =\left(\begin{array}{c|ccc}
* & & * & \\
* & 0 & & * \\
* & & 0 & \\
* & & \cdot
\end{array}\right)+\left(\begin{array}{ll}
0 & 0 \\
0 & \varepsilon
\end{array}\right) .
\end{aligned}
$$

This implies that $T$ is a norm limit of zero-diagonal operators. Indeed, the first term of the last equality can be written as
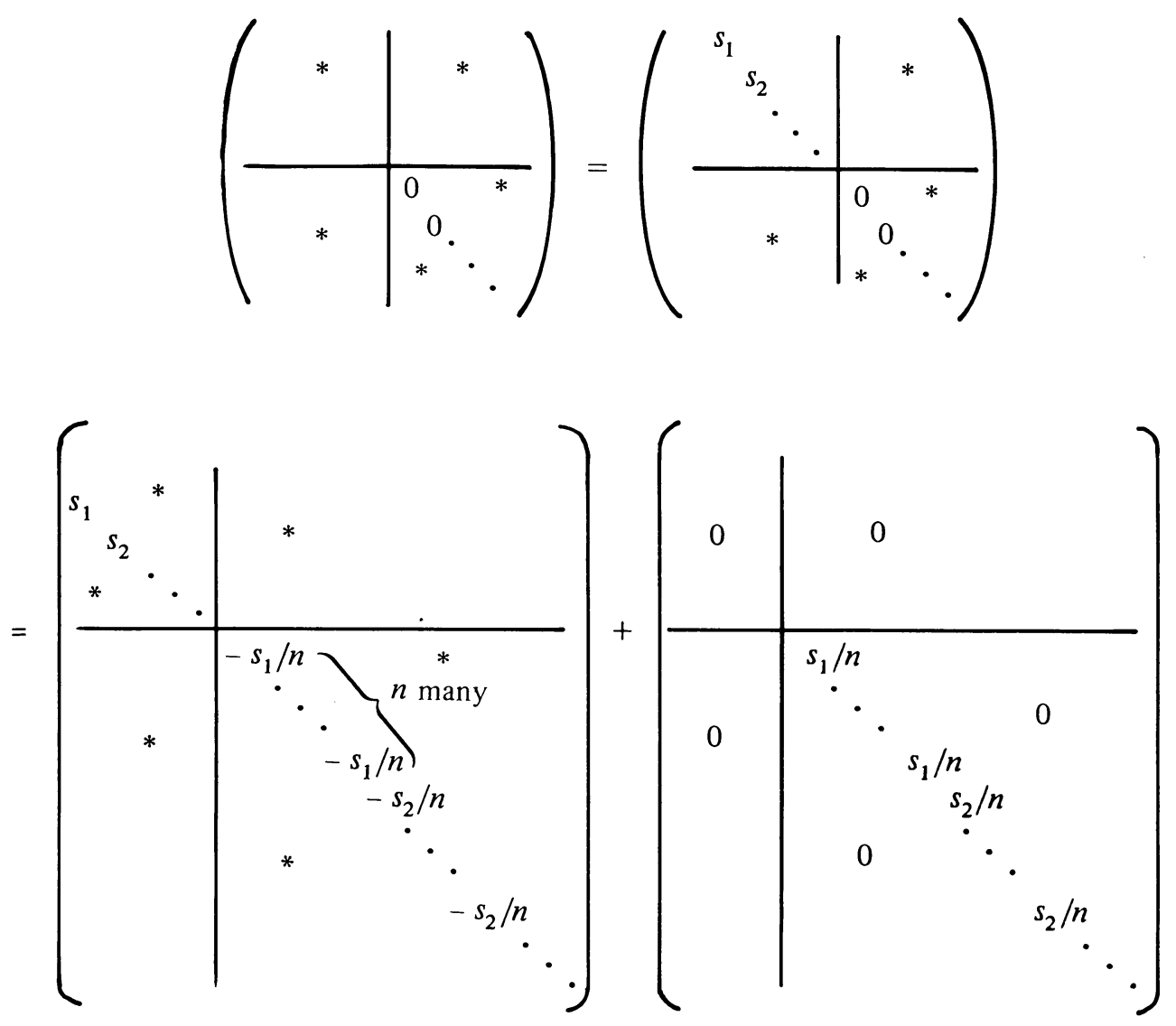
by Theorem 1, the first term to the right of the second equality is a zero-diagonal operator (the diagonal entries can be rearranged) and the norm of the second term is $\leqslant\|T\| / n$. These complete the proof of Theorem 3 .

REMARK. By [6, Theorem (5.1)], the following statements are also necessary and sufficient conditions for an operator $T$ being a norm limit of zero-diagonal operators:

(1) $0 \in \cap\left\{W(T+F)^{-}: F\right.$ is of finite rank $\}$.

(2) There exists a sequence $\left\{x_{n}\right\}$ of unit vectors such that $x_{n} \rightarrow 0$ weakly and $\left(T x_{n}, x_{n}\right) \rightarrow 0$.

(3) There exists an orthonormal sequence $\left\{e_{n}\right\}$ such that $\left(T e_{n}, e_{n}\right) \rightarrow 0$.

(4) There exists an infinite-dimensional projection $P$ such that $P T P$ is compact.

For other equivalent conditions, the reader is referred to Anderson [1] and Stout [11].

COROLlaRy. $T$ is the norm-closure of $\lambda$-diagonal operators if and only if $\lambda \in W_{e}(T)$.

Since $W_{e}(T) \neq \varnothing$, this corollary implies that every operator can be approximated uniformly by operators unitarily equivalent to an infinite matrix with constant main diagonal; thus it extends a result by P. Fillmore [4], which states that every operator acting on a finite dimensional space is unitarily equivalent to an operator with constant diagonal entries. Applying Theorem 3 one can find out about the size of zero-diagonal operators.

COROLlARY. The set of zero-diagonal operators is of second category, but not dense in $B(H)$.

Proof. To show that this set is not of first category, it is enough to show it contains an open set. Put $T=I \oplus(-I) \oplus i I \oplus(i I)$. It is clear that $W_{e}(T)=$ the convex hull of $\{1, i,-1,-i\}$. Thus $0 \in W_{e}(T+S)$ for $\|S\|<\frac{1}{4}$. In other words, $T$ is in the interior of the set.

It is trivial to see that this set is not dense in $B(H)$.

We now answer the question as to whether the set of zero-diagonal operators is closed under similarity. Unfortunately, the answer is negative in infinite-dimensional setting (though affirmative for trace class operators). In the following we shall construct similar operators $S$ and $T$ such that $0 \notin W_{e}(S)$, but $0 \in W_{e}(T)$. We shall then produce a zero-diagonal operator that is similar to a nonzero-diagonal one.

Take

$$
A_{j}=\left(\begin{array}{ll}
1 & 0 \\
0 & 2
\end{array}\right), \quad B_{j}=\left(\begin{array}{ll}
1 & 3 \\
0 & 2
\end{array}\right), \quad \text { and } \quad Q_{j}=\left(\begin{array}{cc}
1 & -3 \\
0 & 1
\end{array}\right) ;
$$

then $A_{j}=Q_{j} B_{j} Q_{j}^{-1}, W\left(A_{j}\right)=[1,2]$, and $W\left(B_{j}\right) \supseteq[0,2]$. Let $S=\Sigma \oplus A_{j}, T=$ $\Sigma \oplus B_{j}$, and $Q=\Sigma \oplus Q_{j}$; clearly, $W_{e}(S)=[1,2], W_{e}(T) \supseteq[0,2]$, and $S=Q T Q^{-1}$. We thus have similar operators such that $0 \notin W_{e}(S)$ but $0 \in W_{e}(T)$. Because $0 \in W_{e}(T)$, by Theorem 3 there exists a sequence of zero-diagonal operators $T_{n} \rightarrow T$. Since

$$
\left\|Q T_{n} Q^{-1}-S\right\|=\left\|Q\left(T_{n}-T\right) Q^{-1}\right\| \leqslant\|Q\| \cdot\left\|Q^{-1}\right\| \cdot\left\|T_{n}-T\right\| \rightarrow 0
$$


it follows that $Q T_{n} Q^{-1}$ is not a norm-limit of zero-diagonal operators for big enough $n$. So $T_{n}$ and $Q T_{n} Q^{-1}$ fulfill our assertion for such $n$ 's.

We now focus our attention on hermitian operators. In [9] H. Radjavi showed that, assuming $T$ is hermitian, the following statements are equivalent:

(1) $T=A^{*} A-A A^{*}$, where $A \in B(H)$.

(2) $T=2 i\left(A_{1} A_{2}-A_{2} A_{1}\right)$, where $A_{1}$ and $A_{2}$ are hermitian.

(3) There exists an orthonormal sequence $\left\{e_{j}\right\}$ of vectors such that $\sum_{j=1}^{n}\left(T e_{j}, e_{j}\right)$ is bounded.

(4) $\mathrm{Sp}(T)$ has at least one nonnegative limit point and at least one nonpositive limit point.

(5) $T$ is not of the form $T_{1} \oplus T_{2}$, where $T_{1}$ has finite-dimensional domain and where either $T_{2} \geqslant a>0$ or $T_{2} \leqslant a<0$ for some real number $a$.

Also equivalent to the above statements is the following [5]:

(6) $T$ is the real part of a quasinilpotent operator.

Of course, statement (4) is equivalent to saying that $0 \in W_{e}(T)$. In what follows, we are to provide two new descriptions for self-commutators, inspired by the fact that if $H$ is finite-dimensional, the following statements are equivalent:

(1) $T=A^{*} A-A A^{*}$, where $A \in B(H)$;

(2) $\operatorname{Tr}(T)=0$;

(3) $T$ has a zero-diagonal.

THEOREM 4. Let $T$ be a (bounded) hermitian operator. Then the following are equivalent:

(1) $T=A^{*} A-A A^{*}$, where $A \in B(H)$;

(2) $0 \in W_{e}(T)$;

(3) $T$ is a norm limit of zero-diagonal operators;

(4) $T$ is a norm limit of hermitian operators $S$, with $\operatorname{Tr}\left(S^{+}\right)=\operatorname{Tr}\left(S^{-}\right) \leqslant \infty$.

Proof. (1) $\Leftrightarrow(2)$, by the above-mentioned Radjavi's theorem. (2) $\Leftrightarrow(3)$, by a slight modification of Theorem 3. (3) $\Leftrightarrow$ (4), by Theorem 2 of $\S 1$. These complete the proof.

The following result is a direct consequence of the above-mentioned Radjavi's theorem.

THEOREM 5. Let $T$ be a self-commutator and write $T=T^{+} \oplus\left(-T^{-}\right)$with respect to $\mathrm{H}^{+} \oplus \mathrm{H}^{-}$, where $\mathrm{T}^{+}$and $\mathrm{T}^{-}$are positive definite on $\mathrm{H}^{+}$and $\mathrm{H}^{-}$, respectively. Then $T$ is invertible if and only if $T^{+}$and $T^{-}$are invertible and $\operatorname{dim} H^{+}=\infty=\operatorname{dim} H^{-}$.

As immediate corollary, we list the following fact (see, e.g., [8, p. 132]).

Corollary [PUTNam]. A positive self-commutator cannot be invertible.

Combining Theorems 2 and 5, we may have a surprising result.

THEOREM 6. Suppose $T$ is hermitian and invertible. Then $T$ has a zero-diagonal if and only if $T^{-1}$ has one. (Observe that this is not true if $T$ is a matrix.) 
But this theorem no longer holds if $T$ is not hermitian. Indeed, take $T=$ $\operatorname{diag}\{i, i,-2 i ; 1,1, \ldots ;-1,-1, \ldots\}$; then $T$ has a zero-diagonal, but $T^{-1}$ does not have one. However, a weaker form of this theorem does hold.

THEOREM 7. Let $T$ be an invertible operator. Then $0 \in W_{e}(T)$ if and only if $0 \in W_{e}\left(T^{-1}\right)$.

Proof. By Theorem 3 of $\S 2$, there exists a sequence of invertible zero-diagonal operators $\left\{T_{n}\right\} \rightarrow T$, in the norm topology; and hence, $\left\{T_{n}^{-1}\right\} \rightarrow T^{-1}$, in the norm topology. Thus, again by Theorem 3 it is enough to show that $0 \in W_{e}\left(T_{n}^{-1}\right)$ for $n=1,2, \ldots$

To that end, let $\left\{e_{j}\right\}$ be an orthonormal basis such that $\left(T_{n} e_{j}, e_{j}\right)=0$; this implies $\left(f_{j}, T_{n}^{-1} f_{j}\right)=0$ and thus $\left(g_{j}, T_{n}^{-1} g_{j}\right)=0$, where $f_{j}=T_{n} e_{j}$ and $g_{j}=f_{j} /\left\|f_{j}\right\|$. Now it is sufficient to prove that there exists a subsequence $\left\{g_{n,}\right\}$ of $\left\{g_{j}\right\}$ so that $\left\{g_{n}\right\} \rightarrow 0$ weakly. Put $g_{n_{1}}=g_{1}$. Since

$$
\left\|T_{n}^{*} T_{n} e_{1}\right\|^{2}=\sum_{j}\left|\left(T_{n}^{*} T_{n} e_{1}, e_{j}\right)\right|^{2}
$$

it follows that

$$
\left(f_{1}, f_{j}\right)=\left(T_{n} e_{1}, T_{n} e_{j}\right)=\left(T_{n}^{*} T_{n} e_{1}, e_{j}\right) \rightarrow 0 \text { as } j \rightarrow \infty,
$$

hence

$$
\left(g_{1}, g_{j}\right)=\frac{\left(f_{1}, f_{j}\right)}{\left\|f_{1}\right\| \cdot\left\|f_{j}\right\|}=\frac{\left(f_{1}, f_{j}\right)}{\left\|T_{n} e_{1}\right\| \cdot\left\|T_{n} e_{j}\right\|} \leqslant\left(f_{1}, f_{j}\right) \cdot\left\|T_{n}^{-1}\right\|^{2} \rightarrow 0 \cdot\left\|T_{n}^{-1}\right\|^{2}=0
$$

as $j \rightarrow \infty$.

Set $g_{n_{2}}=g_{j}$ so that $\left|\left(g_{n_{1}}, g_{n_{2}}\right)\right| \leqslant 1 / 2^{2}$. In the same fashion, we can manufacture $g_{n_{3}}$, $g_{n_{4}}, \ldots$ such that $\left|\left(g_{n_{k}}, g_{n_{l}}\right)\right| \leqslant 1 / 2^{m}$, where $m=\max (k, l)$. Clearly $\left\{g_{n_{j}}\right\} \rightarrow 0$ weakly. This completes the proof of the theorem.

Before closing this section, we would like to characterize a seemingly unrelated class of operators-trace class operators.

THEOREM 8. Let $T$ be an operator. If for every orthonormal basis there exists a rearrangement $\left\{b_{j}\right\}_{j=1}^{\infty}$ so that a subsequence $\left\{s_{n_{k}}\right\}_{k=1}^{\infty}$ of $\left\{s_{n}\right\}_{n=1}^{\infty}$ converges, where $s_{n}=\sum_{j=1}^{n}\left(T b_{j}, b_{j}\right)$, then $T$ is a trace class operator.

Proof. Write $T=T_{1}+i T_{2}$. We claim that $W_{e}\left(T_{1}\right)=\{0\}$. Indeed, if $0 \neq \lambda \in$ $W_{e}\left(T_{1}\right)$, say $\lambda>0$, then, by a corollary of Theorem 3 , there exists an orthonormal basis $\left\{e_{j}\right\}$ such that $\left(T_{1} e_{j}, e_{j}\right) \geqslant \lambda / 2>0$ for all $j$. This clearly violates the hypothesis.

Since $\operatorname{Sp}_{e}\left(T_{1}\right)=$ convex hull $W_{e}\left(T_{1}\right)=\{0\}$ (see [10]), it follows that $T_{1}$ is compact, so is $T_{2}$. Hence $T$ is compact. We now apply Theorems 3 and 4 to conclude that $T_{1}$ and $T_{2}$ are trace class operators. (In fact, $\Sigma p_{i}$ and $\Sigma q_{j}$ must be finite, where $\left\{p_{i}\right\}$ and $\left\{-q_{j}\right\}$ are the nonnegative and the negative eigenvalues of $T_{1}$ respectively.) 


\section{REFERENCES}

1. J. Anderson, Derivations, commutators and the essential numerical range, Ph.D. Thesis, Indiana University, 1971.

2. A. Brown and C. Pearcy, Structure of commutators of operators, Ann. of Math. (2) 82 (1965), $112-127$.

3. P. Fan and C. K. Fong, Which operators are the self-commutators of compact operators? Proc. Amer. Math. Soc. 80 (1980), 58-60.

4. P. Fillmore, On similarity and the diagonal of a matrix, Amer. Math. Monthly 76 (1969), 167-169.

5. P. Fillmore, C. K. Fong and A. Sourour, Real parts of quasinilpotent operators, Proc. Edinburgh Math. Soc. 22 (1979), 263-269.

6. P. Fillmore, J. Stampfli and J. Williams, On the essential numerical range, the essential spectrum and a problem of Halmos, Acta Sci. Math. (Szeged) 33 (1972), 179-192.

7. P. R. Halmos, Finite-dimensional vector spaces, Van Nostrand, Princeton, N.J., 1958.

8. __ A Hilbert space problem book, Van Nostrand, Princeton, N.J., 1967.

9. H. Radjavi, Structure of $A^{*} A-A A^{*}$, J. Math. Mech. 16 (1966), 19-26.

10. J. G. Stampfli and J. P. Williams, Growth conditions and the numerical range in a Banach algebra, Tôhoku Math. J. 20 (1968), 417-424.

11. Q. F. Stout, Schur products of operators and the essential numerical range, Trans. Amer. Math. Soc. 264 (1981), 39-47.

Department of Mathematics, Marshall University, Huntington, West Virginia 25701

Current address: Department of Mathematics, Texas Christian University, Fort Worth, Texas 76129 